\title{
Efecto de la acción posterior a la recepción sobre la amortiguación de los saltos verticales
}

\author{
Accésit Congreso SIBB 2016
}

M. GUTIÉRREZ-DAVILA, J. OLIVARES, D. PANCORBO, F.J. ROJAS

Departamento de Educación Física y Deportiva. Universidad de Granada

\begin{abstract}
Resumen
El principal objetivo de este estudio ha sido comprobar el efecto que tienen tres acciones posteriores al aterrizaje del salto vertical (aterrizaje discreto (AD), aterrizaje preparatorio al salto $(A P S)$, aterrizaje preparatorio a la carrera $(A P C)$ ) sobre los factores mecánicos que determinan la amortiguación. Han participado 29 estudiantes con experiencia en deportes donde el salto vertical constituye una habilidad básica. Se ha utilizado una plataforma de fuerza operando a $500 \mathrm{~Hz}$, sincronizada con una cámara de vídeo que registraba a $210 \mathrm{~Hz}$ el plano sagital del movimiento. En la situación $A D$, debían dejarse caer a una plataforma desde una altura de $0.5 \mathrm{~m}$ partiendo de una posición erguida con los brazos por encima de la cabeza y permitiendo su participación libre durante la fase de impacto (FI). En la condición APS, se procede igual pero, a continuación, se realiza un salto vertical máximo y, en la condición APC, el aterrizaje se acompaña con un desplazamiento horizontal. Los resultados indican que el 2-PMF (pico máximo de fuerza) es mayor para AD, alcanzándose antes que en APS y APC. Al inicio de la recepción el centro de gravedad está más adelantado en $A P C$ debido a la carrera posterior. En el 2-PMF, el desplazamiento horizontal del CG (SxCG) y la velocidad horizontal del $C G(V x C G)$ se han incrementado con la carrera posterior con respecto a las otras dos situaciones, una tendencia cinemática que se mantiene hasta el final de FI. Con la carrera posterior, la mayor contribución a la amortiguación la realizan las piernas en su desplazamiento hacia delante, mientras que en las otras dos situaciones se compensa con el desplazamiento hacia atrás de los muslos.
\end{abstract}

Palabras clave: Biomecánica, Aterrizaje salto vertical, Amortiguación, Lesiones deportivas.

\begin{abstract}
The main aim of this research has been to verify the effect, that three actions after the landing of the vertical jump (discrete landing (AD), preparatory landing to the jump (APS), and preparatory landing to the race (APC)), on the mechanical factors that determine the jump's damping. Twenty-nine students with experience in sports where vertical jumping is a basic skill. A force platform operating at $500 \mathrm{~Hz}$, synchronized with a video camera recording the sagittal plane of the motion at $210 \mathrm{~Hz}$ were used. In AD condition, the participants dropped onto a platform from a height of $0.5 \mathrm{~m}$ starting with the arms above the head and allowing their free participation during the impact phase (FI). In the APS condition, the same procedure was followed, but after the landing, a maximum vertical jump was made and in the APC condition the landing was accompanied by an immediate run. The results indicate that second peak of force is higher for AD, reaching it before than in APS and APC conditions. At the start of the reception, the CG is more advanced in APC due to the later race. In the second peak of force, the horizontal displacement and have increased with the posterior run with respect to the other two situations, a kinematic tendency that remains until the end of FI. In the APC situation, the greatest contribution to the damping is made by the calves in their forward movement, while in the other two situations it is compensated by the backward movement of the thighs.
\end{abstract}

Keywords: Biomechanics, Vertical jump landing, Damping, Sports injuries.

Correspondencia:

Marcos Gutiérrez Dávila

Universidad de Granada. Departamento de Educación Física y Deportiva

Carretera de Alfacar s/n (18071 - Granada, España)

e-mail: marcosgd@ugr.es 


\section{Introducción}

El salto es una habilidad básica presente en numerosas modalidades deportivas cuyo aterrizaje posee fines diferentes. En este sentido, Caster [2] distingue dos grupos: a) Aterrizajes discretos, considerados como los que se producen posteriores a la acción, solo con el objetivo de amortiguar la caída y b) Aterrizajes preparatorios, como aquellos que se producen antes de una acción posterior determinada y tienen como objetivo activar el ciclo estiramiento acortamiento, o producir un impulso de frenado que permita incrementar el impulso de aceleración posterior.

La mayor parte de los trabajos de investigación dirigidos hacia la prevención de lesiones se han centrado en los aterrizajes discretos [4, 6 , 27], mientras que el análisis de los aterrizajes preparatorios se ha orientado más hacia el rendimiento de acciones posteriores [18, $20,26]$. Por lo tanto, son escasas las investigaciones que han tratado los aterrizajes preparatorios desde una perspectiva del análisis de la absorción de las fuerzas de reacción con el propósito de tratar de dar explicación a la génesis de ciertas lesiones deportivas.

En esta investigación se pretende comprobar el efecto que tienen tres acciones posteriores al aterrizaje (aterrizaje discreto, preparatorio al salto y preparatorio a la carrera), sobre los factores mecánicos que determinan la amortiguación. Decker [6] comprobó que las extremidades inferiores reducen y controlan la aceleración descendente adquirida durante la fase de vuelo mediante la flexión de sus articulaciones de tal manera que reducen las fuerzas de reacción mediante una ligera flexión en el contacto inicial con el suelo, seguida de una gran amplitud de movimiento articular tras el mismo.

Los movimientos de las extremidades inferiores para reducir las fuerzas de reacción pueden estar mediatizados por las acciones posteriores a realizar por los deportistas. En este sentido, Zhang [32] considera que las alteraciones en las posiciones corporales y velocidades angulares durante el aterrizaje podrían influir en las magnitudes de los momentos de fuerza que producen tensión en la estructura interna de la rodilla, lo que adquiere especial relevancia cuando la articulación de la rodilla se considera como el principal amortiguador en la recepción [6].

Apoyando esta teoría, Kuling et al. [22], utilizando movimientos de danza donde la demanda estética condiciona en gran medida la amortiguación de los saltos, han puesto de manifiesto que dicha demanda limitaría la variabilidad individual de ejecución técnica, e incrementaría la rigidez articular durante la recepción. Dos aspectos relacionados y que se asocian al riesgo de producirse lesiones.

De la misma forma, DeVita y Skelly [7], mediante la comparación de aterrizajes rígidos y aterrizajes suaves desde una altura de 59 $\mathrm{cm}$, comprobaron que la rigidez articular de la rodilla en el momento de la recepción incrementaba las fuerzas de reacción del suelo, aumentando, en consecuencia, el riesgo de lesión.

Por otro lado, ciertas lesiones deportivas también se relacionan con el aumento del pico máximo de las fuerzas de reacción procedentes del suelo durante la amortiguación [2]. Entre las lesiones sin contacto más graves que se atribuyen a los aterrizajes de saltos, una de las más comunes es la rotura de ligamento cruzado anterior (LCA) $[1,10,11,12,14,24]$.

Aunque no existen datos concluyentes sobre la influencia que tienen las demandas posteriores a la recepción sobre el riesgo de lesión, las aportaciones expuestas parecen evidenciar que las exigencias de ciertas acciones posteriores al aterrizaje podrían incrementar la tensión en estructuras internas de las articulaciones. Por el contrario, ciertas teorías procedentes de la neuromecánica muscular ponen de manifiesto que, cuando se realiza un aterrizaje preparatorio para realizar un segundo salto, se incrementa la activación neuromuscular antes de iniciarse la toma de contacto, lo que predispone al sistema músculo esquelético para iniciar la absorción del impacto con mayor eficacia, reduciendo así el impacto inicial [13, 21]. Mizrahi [25], corroborando esta teoría, destaca la importancia de mejorar los ajustes de preactivación muscular no reflejos en la primera fase de impacto con el fin de reducir las fuerzas de reacción del suelo. 
Según las evidencias y controversias expuestas que se derivan de las investigaciones precedentes, se plantea como hipótesis que la acción posterior al aterrizaje reduce las fuerzas de reacción del suelo durante la amortiguación y condiciona el modelo de ejecución técnica, pudiendo situar ciertos segmentos en posiciones que incrementan los momentos de fuerza que pueden soportar las articulaciones asociadas.

\section{Método}

\section{Participantes}

Han participado 29 estudiantes (hombres) de Ciencias de la Actividad Física y del Deporte $($ edad $=21,1 \pm 1,7$ años; talla $=1,78 \pm 0,06$ $\mathrm{m}$; masa $=70.6 \pm 8,1 \mathrm{~kg}$ ), utilizando para su selección el criterio de haber participado en deportes donde sea habitual la realización de saltos. A todos ellos se les informó y solicitó su consentimiento para participar en este estudio, siguiendo las directrices de la Comisión Ética de la Universidad de Granada.

\section{Material y procedimientos}

Se ha utilizado una plataforma de fuerza Dinascan IBV, operando a $500 \mathrm{~Hz}$, sincronizada temporalmente a una cámara de vídeo Casio EXFH20, que registraba a $210 \mathrm{~Hz}$ el plano sagital del movimiento.

Después de un calentamiento normalizado de 10 minutos basado en carrera y estiramientos, y un proceso de adaptación a las tres condiciones experimentales en el cual se practicaron cada una de ellas durante un tiempo aproximado de 20 minutos, se realizaron cinco ensayos para cada condición: en la condición aterrizaje discreto $(\mathrm{AD})$, los participantes debían dejarse caer a la plataforma desde una altura de 0.5 metros, partiendo de una posición erguida con los brazos por encima de la cabeza y permitiendo su participación libre durante la fase de vuelo y amortiguación de la caída. En el aterrizaje preparatorio al salto (APS), se parte de la misma posición anterior, aunque en este caso el participante debe realizar un salto máximo inmediatamente después de la amortiguación. Finalmente, en el aterrizaje preparatorio a la carrera (APC), se proce- de igual que en el anterior, pero el propósito es realizar un rápido desplazamiento horizontal tras la recepción. El orden de las condiciones se cambió para cada participante.

\section{Análisis de los datos}

Para el análisis posterior se seleccionó el ensayo cuyo tiempo de absorción era el mediano entre los cinco de cada situación experimental. Este tiempo de absorción se definió como el periodo comprendido desde el inicio de la recepción hasta el instante en que la velocidad vertical del centro de gravedad (CG) alcanzaba valores positivos. A partir de los datos procedentes de la plataforma se registró el valor máximo del segundo pico máximo de fuerza (2-PMF), así como los registros de las dos componentes de la velocidad del CG y sus respectivos desplazamientos durante la fase de impacto, la cual estaba definida por los primeros $100 \mathrm{~ms}$ desde el contacto con la plataforma [28]. Para su determinación se utilizó dinámica inversa, siguiendo el método descrito por Gutiérrez-Dávila et al. [15].

Las constantes de integración se han obtenido a partir de la digitalización manual de las imágenes de vídeo, utilizando para ello un modelo mecánico plano de 8 segmentos, determinados por 11 puntos (extremos anterior y posterior del pie; centros articulares del tobillo, rodilla, cadera, codo y muñeca; tercer metacarpiano de la mano; hueco supraexternal; ángulo del maxilar y vertex), donde las masas segmentarias y las respectivas localizaciones de sus centros de masas (CM) se han obtenido a partir de los valores propuestos por Zatsiorsky \& Seluyanov (1983) y adaptados por de Leva, [31].

Tras la digitalización, las coordenadas planas fueron suavizadas mediante un filtro digital de paso bajo, a $8 \mathrm{~Hz}$ [29] e interpoladas a $500 \mathrm{~Hz}$, utilizando splines de quinto grado. La conversión de las coordenadas digitalizadas en datos reales se realizó mediante un sistema de referencia que consistía en un cubo de $2 \times 2 \times 0.5$. La constante de integración correspondiente a la velocidad instantánea en el momento aproximado de la toma de contacto con la plataforma, se determinó mediante la primera derivada de la posición vertical con 
respecto al tiempo, utilizando las funciones Quintic splines [30]. A partir de las coordenadas planas del modelo, se determinaron las posiciones angulares de las articulaciones de la cadera, rodilla y tobillo, utilizando para ello el producto escalar de los vectores que definían las posiciones de sus respectivos segmentos. Es necesario aclarar que el segmento pie se definió como el vector que une los puntos del centro articular del tobillo y el final del pie. Finalmente se determinó la contribución de los brazos, tronco+cabeza, muslos, piernas y pies a la distancia vertical de frenado del CG durante la fase de impacto, utilizando para ello el método de cálculo propuesto por GutiérrezDávila et al [16].

\section{Resultados}

En la tabla 1 se presenta la estadística descriptiva e inferencial de las variables generales relacionadas con la amortiguación de la caída para las tres condiciones experimentales. Los datos indican que han existido diferencias significativas $(\mathrm{p}<0.01)$ en el pico máximo de fuerza (2-PMF) entre las medias de las 3 condiciones experimentales expuestas, expresado ese dato en relación al peso corporal de cada participante. La prueba de contrastes aplicada a esta variable indica que los aterrizajes discretos producen un pico de fuerza mayor que cualquiera de las otras dos situaciones de aterrizaje preparatorios. El tiempo en el que se alcanza el 2-PMF ( $\left.\mathrm{t}_{(2-\mathrm{PMF})}\right)$, ha sido mayor en los aterrizajes preparatorios al salto, con respecto a las otras dos condiciones $(\mathrm{p}<0.01)$. Con respecto a la localización del CG en el momento de la toma de contacto con la plataforma, éste se encuentra significativamente más alto (Y $\mathrm{YG}_{\mathrm{CG}}$ (TOMA CONTACTO); $\mathrm{p}<0.05$ ) cuando el aterrizaje es discreto, con respecto a las otras dos condiciones. En cuanto a la componente horizontal, el CG está más adelantado cuando el aterrizaje es preparatorio a la carrera (X $\mathrm{XG}_{\mathrm{CG} \text {-(TOMA CONTAC- }}$ то); $\mathrm{p}<0.001$ ). En la misma tabla se muestra la posición angular de la articulación de la cadera en el instante de la toma de contacto con la plataforma. Los datos ponen de manifiesto que han existido altas diferencias entre las medias ( $\left.\theta_{\text {CADERA-(TOMA CONTACTO); }} \mathrm{p}<0.001\right)$, siendo menor el ángulo medio en la condición de aterrizaje preparatorio al salto, con respecto a los otros dos.

En la tabla 2 se presenta una estadística descriptiva e inferencial de las componentes del desplazamiento del CG en la fase de impacto que, como se había descrito, se produce durante los 100 ms de la toma de contacto. Los datos ponen de manifiesto que existe una cierta significación en el desplazamiento vertical del CG (SyCG(100) $\mathrm{p}<0.05$ ), aunque la prueba de contrastes solo nos permite afirmar que éste

\begin{tabular}{|c|c|c|c|c|}
\hline Variables & Discreto (AD) & $\begin{array}{l}\text { Preparatorio } \\
\text { al salto (APS) }\end{array}$ & $\begin{array}{l}\text { Preparatorio a } \\
\text { la carrera (APC) }\end{array}$ & $\boldsymbol{F}$ \\
\hline 2-PMF $(N / N)$ & $4.941 \pm 1.344$ & $4.157 \pm 1.211^{1}$ & $3.936 \pm 0.886^{1}$ & $13.17^{\star *}$ \\
\hline$t_{(2-\mathrm{PMF})}(s)$ & $0.050 \pm 0,015$ & $0.054 \pm 0,015$ & $0.066 \pm 0,020^{1,2}$ & $9.79^{* *}$ \\
\hline$Y_{\text {CG-(TOMA CONTACTO) }}(m)$ & $1.154 \pm 0,041$ & $1.136 \pm 0,048^{1}$ & $1.132 \pm 0,053^{1}$ & $3.35^{*}$ \\
\hline $\boldsymbol{X}_{\text {CG-(TOMA CONTACTO) }}(\mathrm{m})$ & $-0.068 \pm 0,018$ & $-0.042 \pm 0,029$ & $0.110 \pm 0,078^{1,2}$ & $111.27^{\star * *}$ \\
\hline$\theta_{\text {TOBILLO-(TOMA CONTACTO) }}\left({ }^{\circ}\right)$ & $134 \pm 7$ & $132 \pm 11$ & $130 \pm 8$ & 2.93 \\
\hline$\theta_{\text {RODILLA-(TOMA CONTACTO) }}\left({ }^{\circ}\right)$ & $159 \pm 6$ & $154 \pm 7^{1}$ & $157 \pm 7$ & $4.05^{\star}$ \\
\hline$\theta$ CADERA-(TOMA CONTACTO) $\left({ }^{\circ}\right)$ & $157 \pm 6$ & $151 \pm 8^{1}$ & $159 \pm 9^{2}$ & $11.83^{\star \star *}$ \\
\hline
\end{tabular}

Tabla 1. Estadística descriptiva e inferencial de las variables generales relacionadas con la amortiguación de la caída para las tres condiciones experimentales: Los valores de la fuerza se han expresado en Newton de fuerza por Newton de peso corporal. 
es mayor cuando la recepción se realiza para la preparación del salto vertical con respecto de las otras 2. Sin embargo, el mayor desplazamiento horizontal del CG cuando se prepara a la carrera $\left(\mathrm{Sx}_{\mathrm{CG}(100)} \mathrm{p}<0.01\right)$, reduce las diferencias de los desplazamientos resultantes entre los dos aterrizajes preparatorios. Algo similar ocurre con las componentes de la velocidad al final de la fase de impacto. Con respecto a la componente vertical existe una alta significación entre las medias ( $\left.\mathrm{vy}_{\mathrm{CG}(100)}, \mathrm{p}<0.001\right)$, siendo sus valores negativos superiores para la condición de aterrizaje preparatorio al salto con respecto a las otras dos condiciones. Aunque, como sucedía con el desplazamiento del CG, la mayor velocidad horizontal del CG cuando se prepara la carrera $\left(\mathrm{vx}_{\mathrm{CG}(100)}\right.$, $\mathrm{p}<0.001)$ hace que se reduzcan las diferencias de las velocidades resultantes entre los dos aterrizajes preparatorios.

En la tabla 3 se presenta el análisis de la contribución segmentaria para las tres condiciones. En ella se pueden apreciar las contribuciones de los brazos, tronco+cabeza, muslos, piernas y pies, expresadas en porcentajes del desplazamiento vertical del CG hasta el final de la fase de impacto. Se observa que, para los tres tipos de aterrizaje, la mayor contribución la realizan las piernas. La estadística inferencial indica que esta contribución es significativamente mayor cuando la recepción es preparatoria de la carrera con respecto a las otras dos $(\mathrm{p}<0.001)$, mientras que la del muslo se reduce de forma muy significativa $(\mathrm{p}<0.001)$ compensándose, en cierto modo, con una mayor contribución del tronco+cabeza $(\mathrm{p}<0.01)$.

\begin{tabular}{|c|c|c|c|c|}
\hline Variables & Discreto (AD) & $\begin{array}{c}\text { Preparatorio } \\
\text { al salto (APS) }\end{array}$ & $\begin{array}{c}\text { Preparatorio a } \\
\text { la carrera (APC) }\end{array}$ & $\mathbf{F}$ \\
\hline$S y_{\mathrm{CG}(100)}(m)$ & $0.248 \pm 0,023$ & $0.262 \pm 0,024^{1}$ & $0.250 \pm 0,025^{2}$ & $7.71^{*}$ \\
\hline$S x_{\mathrm{CG}(100)}(m)$ & $0.034 \pm 0,006$ & $0.035 \pm 0,005$ & $0.051 \pm 0,006^{1,2}$ & $34.69^{* *}$ \\
\hline$v \boldsymbol{y}_{\mathrm{CG}(100)}\left(m s^{-1}\right)$ & $-1.746 \pm 0.303$ & $-1.810 \pm 0.287$ & $-1.516 \pm 0.365^{1,2}$ & $14.59^{* * *}$ \\
\hline$v x_{\mathrm{CG}(100)}\left(m s^{-1}\right)$ & $0.171 \pm 0,095$ & $0.223 \pm 0,096$ & $0.755 \pm 0,334^{1,2}$ & $64.07^{* * *}$ \\
\hline${ }^{* * *} p<0.001 ;{ }^{* *} p<0.01 ;{ }^{*} p<0.05$ & \\
\hline
\end{tabular}

Tabla 2. Estadística descriptiva e inferencial de los desplazamientos del CG en el plano sagital en los $100 \mathrm{~ms}$ tras el contacto con la plataforma y las componentes de la velocidad alcanzada por el CG en ese instante.

\begin{tabular}{|l|c|c|c|c|}
\hline \multicolumn{1}{|c|}{ Variables } & $\begin{array}{c}\text { Discreto } \\
(\text { AD) }\end{array}$ & $\begin{array}{c}\text { Preparatorio } \\
\text { al salto (APS) }\end{array}$ & $\begin{array}{c}\text { Preparatorio a } \\
\text { la carrera (APC) }\end{array}$ & $\boldsymbol{F}$ \\
\hline Cbrazos- $_{(100)}(\%)$ & $7.94 \pm 2.74$ & $8.08 \pm 2.61$ & $7.88 \pm 3.12$ & 0.05 \\
\hline Ctronco+cabeza- $_{(100)}(\%)$ & $7.70 \pm 4.04$ & $9.89 \pm 5.39$ & $11.37 \pm 5.51^{1,2}$, & $5.46^{* *}$ \\
\hline${\text { C } \text { muslos- }_{(100)}(\%)}^{(\%)}$ & $29.09 \pm 9.60$ & $27.74 \pm 9.13$ & $10.91 \pm 8.86^{1,2}$ & $45.84^{* \star *}$ \\
\hline Cpiernas- $_{(100)}(\%)$ & $33.04 \pm 8.14$ & $32.56 \pm 8.49$ & $47.00 \pm 13.00^{1,2}$ & $28.57^{* \star *}$ \\
\hline Cpies- $_{(100)}(\%)$ & $23.45 \pm 7.29$ & $23.85 \pm 8.15$ & $23.88 \pm 7.68$ & 0.06 \\
\hline${ }^{* * *} p<0.001 ;{ }^{* *} p<0.01 ;{ }^{*} p<0.05$ & \\
\hline
\end{tabular}

Tabla 3. Estadística descriptiva e inferencial de las contribuciones segmentarias para las tres condiciones experimentales. Los datos se expresan en porcentaje con respecto al desplazamiento del CG en los $100 \mathrm{~ms}$ tras el contacto con la plataforma. 


\section{Discusión}

Los datos han puesto de manifiesto que los aterrizajes discretos producen un pico de fuerza mayor que los aterrizajes preparatorios. La explicación podría estar relacionada con una mayor preactivación muscular debido al ciclo estiramiento-acortamiento en el salto vertical, además del mayor tiempo utilizado para alcanzar el pico de fuerza cuando se utiliza el aterrizaje preparatorio a la carrera. Este dato confirmaría los resultados obtenidos por Caster [2] quien comprobó un incremento de la fuerza máxima de reacción del suelo para los aterrizajes discretos con respecto al preparatorio al salto.

Además, se ha comprobado que el CG en el momento de la toma de contacto con la plataforma está más alto en la condición de aterrizaje discreto, con respecto a los aterrizajes preparatorios. Por lo tanto, la velocidad vertical del CG debería ser ligeramente menor, pero debido al menor tiempo empleado en la amortiguación, el pico de fuerza es mayor. Este comportamiento parece estar relacionado con la mayor extensión de la cadera cuando se compara con el aterrizaje preparatorio al salto, y con el desplazamiento horizontal hacia delante del CG cuando se compara con el aterrizaje preparatorio a la carrera. Esta mayor extensión de la cadera en la recepción podría también explicar el mayor pico de fuerza con respecto a los aterrizajes preparatorios, posiblemente debido a la mayor rigidez articular en la toma de contacto, apoyando la teoría de Kuling y col. [22].

En cuanto a los desplazamientos del CG durante la fase de impacto $(100 \mathrm{~ms})$, se puede afirmar que sus resultantes en los aterrizajes preparatorios son mayores que cuando se realiza el aterrizaje discreto. Esta diferencia, respecto al aterrizaje preparatorio a la carrera, podría confirmar lo indicado por Mizrahi [25], quien sugiere que la acción conjunta de los movimientos y la acción muscular juegan un papel importante en la absorción de fuerzas durante el aterrizaje, traduciéndose ésta en un mayor desplazamiento vertical y hacia delante del CG, incrementando de esta manera el tiempo de frenado de la velocidad vertical.
Con respecto a las componentes de la velocidad del CG al final de la fase de impacto, los datos evidencian un comportamiento similar al obtenido en las del desplazamiento, donde la velocidad resultante del CG es mayor para los aterrizajes preparatorios que para los aterrizajes discretos. En consecuencia, los aterrizajes discretos reducen más la velocidad con menor distancia de frenado del CG, lo que justifica que las fuerzas de reacción durante la fase de impacto sean mayores con respecto a los aterrizajes preparatorios. También se puede afirmar que el modelo de amortiguación de los aterrizajes preparatorios a la carrera es diferente con respecto a los otros dos. Uno de los mecanismos que explican esta diferencia se debe a la estrategia de rotación-extensión a partir de la articulación del tobillo, la cual es necesaria para poder producir fuerzas horizontales al suelo [16] cuando el sujeto pretende iniciar la carrera. Así, se confirma que la acción posterior condiciona la técnica de aterrizaje, modificando las posiciones de los segmentos así como su contribución durante el proceso de amortiguación, lo que podría conducir a incrementar los momentos de fuerza que soportan las articulaciones y, en consecuencia, el riesgo de sufrir lesiones.

En cuanto las contribuciones segmentarias al desplazamiento vertical del CG, resulta interesante destacar la mayor contribución del segmento pierna para las 3 condiciones experimentales. Un dato que estaría de acuerdo con lo aportado por Decker [2], aunque este autor hace referencia a la articulación de la rodilla, y sin embargo nuestros datos indican que la rodilla se flexiona más por el desplazamiento hacia delante de la pierna que por la flexión del muslo. Los datos expuestos señalan, además, un modelo de absorción diferente para los dos aterrizajes preparatorios a la carrera, donde el mayor desplazamiento de la tibia hacia delante junto a la mayor contribución del tronco+cabeza en su flexión hacia delante, incrementaría la tensión de las estructuras de la articulación de la rodilla, lo que se agrava cuando el peso del cuerpo tiende a desplazarse lateralmente hacia el apoyo contrario al que tiene que desplazarse para iniciar la carrera, lo cual ya afirmaron Chappel y col. [3] cuando 
destacaban el aumento de los ángulos de las piernas en valgo como uno de los factores de incremento de la tensión articular.

Este mecanismo podría estar relacionado con ciertas lesiones de ligamento cruzado anterior, ya que su función principal es impedir la traslación anterior de la tibia, coincidiendo con numerosas investigaciones que la han asociado al incremento de las fuerzas durante la recepción de los saltos $[8,19,23]$.

\section{Conclusiones}

Por un lado se ha constatado que se consigue mayor amortiguación de las fuerzas de reacción con los dos tipos de aterrizajes preparatorios, con respecto al aterrizaje discreto, lo que se produce por la menor desaceleración, fundamentalmente por el mayor tiempo utilizado para alcanzar el 2-PMF en APS y APC, y la mayor distancia de frenado del CG.

Por otro, se han identificado cambios en las posiciones de los segmentos durante su contribución a la absorción de las fuerzas en los aterrizajes preparatorios a la carrera, concretamente en el desplazamiento hacia delante y abajo de la tibia con respecto al fémur y el desplazamiento, a su vez, del muslo hacia debajo y del tronco hacia delante, lo que podría incrementar el riesgo de producir lesiones del LCA.

\section{Referencias}

1. Beynnon BD, Vacek PM, Murphy D, Alosa D, Paller D. First-time inversion ankle ligament trauma: The effects of sex, level of competition, and sport on the incidence of injury. Am J Sports Med. 2005;33:1485-91.

2. Caster BL. The effect of height and post-landing movement task on landing performance. Amherst: Biomechanics in Sports XI; 1993. 60-64.

3. Chappell JD, Yu B, Kirkendall DT, Garret WE. A comparison of knee kinetics between male and female recreational athletes in stop-jump tasks. Am J Med. 2002;30(2):261-267.

4. Cortes N, Abrantes J, Gagen L, Dowling E, Onate J, Van Lunen B. Effects of gender and footlanding techniques on lower extremity kinematics during drop-jump landings. J Appl Biomech. 2007; 23:263-268.

5. de Leva $P$. Adjustments to Zatsiorsky-Seluyanovs segment inertia parameters. J Biomech. 1996;29:1223-1230.
6. Decker MJ, Steadman JR, Sterett WI, Torry MR, Wyland DJ. Gender differences in lower extremity kinematics, kinetics and energy absorption during landing. Clin Biomech. 2003;18:662-669.

7. Devita P, Skelly WA. Effect of landing stiffness on joint kinetics and energetics in the lower extremity. Med Sci Sports Exerc. 1992;24(1):108-15.

8. Eriksen HM, Gribble PA, Pfile KR, Pietrosimone BG. Different modes of feedback and peak vertical ground reaction forcé during jump landing. A systematic review. J Athl Training. 2013;48(5):685-682.

9. Ferretti A, Papandrea P, Conteduca F, Mariani PP. Knee ligament injuries in volleyball players. Am J Sports Med. 1992;20:203-207.

10. Ferretti A, Papandrea P, Conteduca F. Knee injuries in volleyball. Sports Med. 1990;10:132-8.

Ferretti A. Epidemiology of jumper's knee. Sports Med. 1986;3:289-95.

11. Ferretti A. Epidemiology of jumper's knee. Sports Med. 1986;3:289-95

12. Frank CB, Jackson DW. Current concepts review: The science of reconstruction of the anterior cruciate ligament. Journal of Bone and Joint Surgery, American Volume. 1997;79:1556-66.

13. Gollhofer A. Kyröläinen H. Neuromuscular control of the human leg extensor muscles in jump exercise under various stretch-load conditions. In J Sports Med. 1991;12:34-40.

14. Griffin LY, Agel J, Albohm MJ, Arendt EA, Dick RW, Garrett WE, et al. Noncontact anterior cruciate ligament injuries: Risk factors and prevention strategies. J Am Acad Orthop Surg. 2000;8:141-150.

15. Gutiérrez-Dávila $M$, Campos J, Navaro E. A comparation of two landing styles in a two-foot vertical Jump. J Strength Cond Res. 2009;23(1):325-331.

16. Gutiérrez-Dávila $M$, Garrido JM, Amaro F, Ramos M, Rojas FJ. Método para determinar la contribución segmentaria en los saltos. Su aplicación en el salto vertical con contramovimiento. Motricidad. Eur J Hum Mov. 2012;29:1-21.

17. Gutiérrez-Dávila $M$, Garrido JM, Amaro FJ, Rojas FJ. Contribución segmentaria en los saltos con contramovimiento en vertical y en horizontal. RICYDE. 2014;28(10):289-304.

18. Gutiérrez-Dávila M, Giles J, Gonzalez C, Gallardo D, Rojas FJ. Efecto de la intensidad del contramovimiento sobre el rendimiento del salto vertical. Apunts. 2015; 119:87-96.

19. Hewett TE, Myer GD, Ford KR, Heidt RS, Colosimo AJ, McLean SG et cols. Biomechanical measures of neuromuscular control and valgus loading on the knee predict anterior cruciate ligament injury risk in female atheletes; A prospective study. Am J Sport Med. 2005;33(4):492-501.

20. Komi PV. Stretch-shortening cycle: a powerful model to study normal and fatigued muscle. J Bio- 
mech. 2000;33(19): 1197-206.

21. Komi PV. Stretch-shortening cycle. Strength and Power in Sport. Blackwell Scientific Publ: Oxford; 1992. 169-179.

22. Kuling PV, Fietzer AL, Popovich JM. Ground reaction forces and knee mechanics in the weight acceptance phase of a dance leap take-off and landing. J Sports Sci. 2011;29(2):125-131.

23. McNair PJ, Marshall RN. Landing characteristics in subjects with normal and anterior cruciate ligament deficient knee joints. Arch Phys Med. 1994;75(5):584-589.

24. Miyasaka KC, Daniel DM, Stone ML, Hirshman P. The incidence of knee ligament injuries in the general population. Am J Knee Surg. 1991;4:3-8.

25. Mizrahi J, Susak Z. Analysis of parameters affecting impact force attenuation during landing in human vertical free fall. Eng Med. 1982;11:141147.

26. Peng $\mathbf{H}$. Changes in biomechanical properties during drop jumps of incremental height. J Strength
Cond Res. 2011; 25(9):2510-2518.

27. Rowley KM, Richards JG. Increasing plantarflexion angle during landing reduces vertical ground reaction forces, loading rates and the hip's contribution to support momento within participants. J Sports Sci. 2015;33(18):1922-1931.

28. Schot PK, Bates BT, Dufek JS. Bilateral performance symmetry during drop landing: a kinetic analysis. Med Sci Sports Exerc. 1994;26:1153-1159.

29. Winter D. Biomechanics and motor control of human movement. New York: John Wiley; 1990.

30. Wood GA, Jennings LS. On the use of spline functions for data smoothing. J Biomech. 1979;12(6):477-9.

31. Zatsiorsky VM, Seluyanov NV. The mass and inertial characteristics of the main segments of the human body. Champaign: Human Kinetics; 1983.

32. Zhang SN, Bates BT, Dufek JS. Contributions of lower extremity joints to energy dissipation during landings. Med Sci Sports Exerc. 2000;32:812-819. 